\title{
Comorbidity of metabolic syndrome components in a population-based screening program: A latent class analysis
}

\author{
Abbas Abbasi-Ghahramanloo ${ }^{1}$, Esmail Moshiri ${ }^{2}$, Sima Afrashteh ${ }^{3}$, Ali Gholami ${ }^{4,5}$, Saeid Safiri ${ }^{6,7}$, \\ Abolfazl Mohammadbeigi*8 (D), Hossein Ansari ${ }^{9}$
}

Received: 7 Apr 2019

Published: 27 Jun 2020

\section{Abstract}

Background: The prevalence of metabolic syndrome (MS) is rapidly increasing in the world. Thus, the aim of the present study was to identify the latent subgroups of Iranian male adults based on MS components and investigate the effect of abnormal alanine aminotransferase (ALT) and aspartate aminotransferase (AST), high total cholesterol (TC), and low-density lipoprotein (LDL) on the odds of membership in each class.

Methods: In the present study, we used the data of a population-based screening program conducted on 823 urban adult men aged 25 years and older in city of Qom in 2014. Abdominal obesity, fasting blood sugar (FBS), blood pressure, and serum lipid profile were measured in participants after for at least 8 hours. MS was defined according to the Adults Treatment Panel III criteria. Latent class analysis was used to achieve the aims of study. Analyses were conducted using PROC LCA in SAS 9.2 software. In all analysis, p value $<0.05$ was considered statistically significant.

Results: There were 3 different latent classes among participants. Latent class 1, non-MS, 55.1\%; latent lass 2, at risk, 21.3\%; and finally latent class 3 , MS, with $23.6 \%$ of the participants. Age (OR=0.98, 95\% CI: $0.98-0.99$, high LDL (OR=0.27, 95\% CI: 0.13-0.56), high TC (OR=8.12, 95\% CI: 4.40-15.00), and abnormal ALT ( $\mathrm{OR}=2.25,95 \%$ CI 1.49-3.41) were associated with at risk class. Also, only age (OR=1.02, 95\% CI: 1.01-1.04) was associated with MS class. The most prevalent components among the participants were having low HDL (34.0\%) and high WC (33.9\%).

Conclusion: Notable percent of samples fell in "at risk" and "MS" classes, which stress the necessity of designing preventive interventions for these specific stratums of population.

Keywords: Metabolic syndrome, Latent class analysis, Subgrouping, Iran

Conflicts of Interest: None declared

Funding: This study was supported by Research Vice-Chancellor of Qom University of Medical Sciences.

\section{*This work has been published under CC BY-NC-SA 1.0 license. \\ Copyright $\odot$ Iran University of Medical Sciences}

Cite this article as: Abbasi-Ghahramanloo A, Moshiri E, Afrashteh S, Gholami A, Safiri S, Mohammadbeigi A, Ansari H. Comorbidity of metabolic syndrome components in a population-based screening program: A latent class analysis. Med J Islam Repub Iran. 2020 (27 Jun);34:69. https://doi.org/10.47176/mjiri.34.69

\section{Introduction}

The prevalence of metabolic syndrome (MS) which is

also called syndrome $\mathrm{X}$, insulin resistance syndrome, and

Corresponding author: Dr Abolfazl Mohammadbeigi, ambeigi@muq.ac.ir

1. Department of Public Health, School of Health, Ardabil University of Medical Sciences, Ardabil, Iran

2. Department of Anesthesiology, Faculty of Medicine, Arak University of Medical Sciences, Arak, Iran

3. Department of Public Health, Faculty of Health, Bushehr University of Medical Sciences, Bushehr, Iran

4. Noncommunicable Diseases Research Center, Neyshabur University of Medical Sciences, Neyshabur, Iran

5. Department of Epidemiology \& Biostatistics, School of Public Health, Neyshabur University of Medical Sciences, Neyshabur, Iran

6. Social Determinants of Health Research Center, Department of Community Medicine, School of Medicine, Tabriz University of Medical Sciences, Tabriz, Iran

7. Aging Research Institute, Tabriz University of Medical Sciences, Tabriz, Iran

8. Research Center for Environmental Pollutants, Department of Epidemiology and Biostatistics, Qom University of Medical Sciences, Qom, Iran

9. Health Promotion Research Center, Department of Epidemiology and Biostatistics, Zahedan University of Medical Sciences, Zahedan, Iran $\uparrow$ What is "already known" in this topic:

The prevalence of metabolic syndrome (MS) is rapidly increasing in the world. In Iran, its prevalence has been reported to be $23.8 \%$ to $36.9 \%$ in adults. Elevated ALT and AST may be risk factors for MS. There is limited information about the relationship of MS with NAFLD.

\section{$\rightarrow$ What this article adds:}

There were 3 different latent classes among participants of this study: Latent class 1, non-MS, 55.1\%; latent lass 2, at risk, $21.3 \%$; and latent class 3 , MS, with $23.6 \%$ of the participants. This study showed that abnormal AST had no significant effect on the membership of adults in different classes. However, abnormal ALT increased the odds of membership in the second class $(\mathrm{OR}=2.25)$. 
etc. is rapidly increasing worldwide $(1,2)$.

The cluster of metabolic factors for MS includes abdominal obesity, high blood pressure, low HDL, high triglyceride levels, impaired fasting glucose, and insulin resistance $(3,4)$. There is evidence that MS greatly increases the risk of developing type 2 diabetes, heart diseases, and mortality rates $(2,5)$. According to the International Diabetes Federation (IDF) reports, about $20 \%-25 \%$ of the world's adult population has MS. Individuals with MS have 2 times greater risk of mortality, 3 times greater risk of heart attack and stroke, and 5 times greater risk of diabetes (6).

Results of previous studies have shown high prevalence of MS in both Western and Asian countries (7-10). For example, the prevalence of MS was reported to be $32.3 \%$, $33 \%$, and $28.3 \%$ in England, India, and Saudi Arabia, respectively (9-11).

MS is a common condition in Iran and is associated with ethnicity, socioeconomic status, and diet. This syndrome can be diagnosed early in adolescence through measuring simple anthropometric indices (12). A number of studies conducted in Iran showed various prevalence of MS among adults ranging from $23.8 \%$ to $36.9 \%$, which is relatively higher than the prevalence of MS among American adults $(3,13,14)$. In a recent study, the overall prevalence of MS was estimated $23.0 \%$, which was the highest in those 55 to 64 years $(38.6 \%)(15)$.

Alanine aminotransferase (ALT) and aspartate aminotransferase (AST) are markers of nonalcoholic fatty liver disease (NAFLD) (16). NAFLD is a component of MS; thus, elevated ALT and AST may be risk factors for MS (17). There is limited information about the relationship of MS with NAFLD.

The clinical definition of MS includes presence of 3 or more of its components. However, it does not matter that a person engages in which of the 3 components $(18,19)$. Understanding which MS components cooccur with each other is possible with subgrouping individuals based on these components (20). Identifying quantitatively and qualitatively different subgroups of MS components is possible with latent class analysis (LCA) (21). Considering distinct profiles of MS can help physicians, health policymakers, and health service providers in applying true decisions in prevention programs $(19,20)$.

The latent class analysis (LCA) is a structural equation model that divides individuals into distinct subgroups with respect to obvious variables. Since the probability of relationships between the components of MS is different in each subgroup, LCA may be useful in describing and identifying the characteristics of these individuals based on the components of MS. In Iran, a few number of studies applied this model to identify the pattern of MS and its components (20). However, this method was never used in previous population-based studies among Iranian adults. Moreover, the effect of abnormal ALT and AST, high total cholesterol (TC), and high LDL on MS components was not evaluated in previous studies. Therefore, the present study aimed to evaluate the pattern of MS by identifying latent classes of MS among Iranian adults and investigate the effect of abnormal ALT and AST, high TC, and high LDL on the odds of membership in each class.

\section{Methods}

\section{Participants}

In the present study, we used the data of a populationbased screening program that was conducted among 823 urban adult men aged 25 years and older in city of Qom in 2014. In this study, participants were selected using multistage random sampling. First, the proportion of each district was calculated to select participants. Next, systematic ransom sampling was used to recruit participants in each stratum. Participants were men aged at least 24 years who were living in Qom, Iran. More details are described in our recent published articles $(15,22)$.

\section{Measurements}

Data collection was designed in 2 stages. At the first stage, a self-repot health interview survey was administrated to evaluate participants' self-report health status and to collect demographic information. In the second stage, based on the study protocol, all participants were invited to take part in screening. A serum lipid profile was ordered for all participants after no caloric intake for at least 8 hours and the laboratory results were assessed. This profile included total cholesterol, high-density lipoprotein (HDL), low-density lipoprotein (LDL), cholesterol, very low-density lipoprotein (VLDL) cholesterol, triglyceride (TG), and hemoglobin tests.

In the present study, MS was defined based on the ATP III (23) criteria. According to this criteria, the presence of 3 or more of the following items considered as MS: (1) high blood pressure $((\geq 130 / 85 \mathrm{mmHg}$ or a history of hypertension), (2) abdominal obesity (waist circumference $>102$ $\mathrm{cm}$ ), (3) $\mathrm{FBG} \geq 110 \mathrm{mg} / \mathrm{dL}$ (or a history of diabetes mellitus), (4) fasting HDL cholesterol $<40 \mathrm{mg} / \mathrm{dL}$, and (5) fasting TGs $\geq 150 \mathrm{mg} / \mathrm{dL}$. The more details of measurements were described in our recent articles $(15,22)$.

\section{Ethical consideration}

All participants in the present study provided written informed consent, and ethical committee of Qom University of Medical sciences approved the study protocol.

\section{Statistical analysis}

In this study, LCA was used for data analysis, which is appropriate for classifying the study participants into different categorical latent variables based on their homogeneity. This analysis assumes that the possible correlation of the indicator variables can be justified by latent variables categories by considering the measurement error. LCA determines the best model with different iterations for the number of identified latent classes and comparing the frequencies of the observed and expected response patterns. For each model, the LCA calculates G2 statistic. Also, Akaike information criterion (AIC) and Bayesian information criterion (BIC) were calculated based on G2 to select the final model (24). For these indices, a smaller value shows a more optimal balance of model fit and parsimony. Finally, a model with the minimum value of AIC or BIC may be selected. 
A. Abbasi-Ghahramanloo, et al.

\begin{tabular}{|c|c|c|c|c|c|c|c|}
\hline $\begin{array}{l}\text { Number of la- } \\
\text { tent class }\end{array}$ & $\begin{array}{c}\text { Number of parameters esti- } \\
\text { mated }\end{array}$ & $\mathrm{G}^{2}$ & $\mathrm{df}$ & AIC & $\mathrm{BIC}$ & P Value & $\begin{array}{c}\text { Maximum } \\
\text { log-likelihood }\end{array}$ \\
\hline 1 & 5 & 313.01 & 26 & 323.01 & 346.57 & $<0.00001$ & -2407.55 \\
\hline 2 & 11 & 77.49 & 20 & 99.49 & 151.33 & $<0.00001$ & -2289.79 \\
\hline 3 & 17 & 20.54 & 14 & 54.54 & 134.66 & 0.114015 & -2261.32 \\
\hline 4 & 23 & 8.48 & 8 & 54.48 & 162.88 & 0.388039 & -2255.29 \\
\hline 5 & 29 & 4.33 & 2 & 62.33 & 199.00 & 0.11475 & -2253.21 \\
\hline
\end{tabular}

Five binary indicator variables were selected to perform LCA, and 5 dichotomous observable variables (eg, indicators) were used to subgroup the participants based on MS components as a latent variable. These indicators were high blood pressure (BP), abdominal obesity, fasting blood sugar (FBS), high-density lipoprotein (HDL), cholesterol, and triglyceride (TG). After finalizing the model, abnormal AST and ALT, high TC, high LDL, and age were entered as covariates in the model.

Given the 5 binary variables, a total of 32 response patterns were identified. Different measures of model assessment are presented in Table 1. Since the degree of freedom of $\mathrm{G}^{2}$ statistic was less than 60 (G2 was distributed approximately as chi-square), the overall significance of the estimated model (Goodness-of-fit) was computed using $\mathrm{G}^{2}$ statistic. When this index is significant, it indicates a significant difference between expected and observed frequencies and, subsequently, it indicates that the fitted model is not appropriate.

The probability of a "No" response can be calculated by subtracting the item-response probabilities from 1 . These probabilities form the basis for interpretation and labeling of latent classes. The larger conditional probabilities appear in bold font to highlight the overall pattern. Due to restrictions of doing complex latent class analysis in SAS software, all analysis was done without considering sampling weights. All analyses were done using PROC LCA in SAS 9.2 software (SAS Institute Inc. Cary, NC, USA). In all analysis, $\mathrm{P}$ value $<0.05$ was considered statistically significant.

\section{Results}

This study was conducted among 823 participants. Findings showed that the mean age of the participants was $54.30 \pm 18.81$ years. Also, $214(26.0 \%)$ of the participants were single. The prevalence of metabolic syndrome components is demonstrated in Table 2. Accordingly, low HDL $(34.0 \%)$ and high WC (33.9\%) were the most prevalent components in the participants.

Based on the results of Table 1, hence, 3, 4 and 5 class models were not significant. In the next stage, the best-fitted model was selected based on G2, AIC, and BIC. The model with the lowest G2, AIC, and BIC values is suitable. According to these model selection indices and interpretability of the results of the model, we concluded that the 3 latent class model was appropriate.

The results of the 3-class model showed that the difference of expected and observed frequency of response pattern was not statistically significant $(\mathrm{G} 2=20.54$, $\mathrm{df}=14$, $\mathrm{p}=0.114$ ). After selecting the final model, we entered age, total cholesterol, LDL, abnormal AST and ALT as covariates in LCA model.

Table 3 shows the results of LCA for the 3-class model. This table has 2 parts: latent class prevalence and item-response probabilities. Latent class prevalence or the probability of the membership in each latent class is presented in the first section of Table 3 .

There are 3 different latent classes among participants. Latent class 1, non-MS, with $55.1 \%$ of the participants; latent lass 2, at risk, with $21.3 \%$ of the participants; and finally latent class 3 , MS, with $23.6 \%$ of participants (Table 3).

Table 2. Percentages of metabolic syndrome (MS) components among Iranian male adults

\begin{tabular}{|c|c|c|c|}
\hline & \multicolumn{3}{|c|}{ Total $(n=823)$} \\
\hline Items & $\mathrm{N}$ & & $95 \% \mathrm{CI}$ \\
\hline High BP & 227 & 27.6 & $25.5-30.6$ \\
\hline High abdominal obesity & 279 & 33.9 & $30.7-37.1$ \\
\hline High FBS & 166 & 20.2 & $17.6-23.1$ \\
\hline Low HDL & 280 & 34.0 & $31.0-37.6$ \\
\hline High TG & 204 & 24.8 & $22.0-28.0$ \\
\hline & \multicolumn{3}{|c|}{ Latent class } \\
\hline & Non-MS & At risk & MS \\
\hline Latent class prevalence & 0.551 & 0.213 & 0.236 \\
\hline \multicolumn{4}{|l|}{ Item-response probabilities } \\
\hline High BP & 0.133 & 0.027 & 0.833 \\
\hline High WC & 0.139 & 0.284 & 0.856 \\
\hline High FBS & 0.092 & 0.135 & 0.522 \\
\hline Low HDL & 0.275 & 0.586 & 0.284 \\
\hline High TG & 0.005 & 0.805 & 0.319 \\
\hline
\end{tabular}

Note: the probability of don't engaging in each components can be calculated by subtracting the item response probabilities shown above from 1 .

$*$ item response probabilities $>0.5$ in bold to facilitate interpretation. 
Table 4. Predictors of membership in latent classes of MS components pattern

\begin{tabular}{lcccc}
\hline Predictors & P value & $\mathrm{C} 1$ & $\mathrm{C} 2$ & $\mathrm{C} 3$ \\
\cline { 3 - 5 } & & OR $(95 \% \mathrm{CI})$ & OR $(95 \% \mathrm{CI})$ & OR $(95 \% \mathrm{CI})$ \\
Age (year) & $<0.001$ & Reference & $0.98(0.96-0.99)$ & $1.02(1.01-1.04)$ \\
High LDL & 0.004 & Reference & $0.27(0.13-0.56)$ & $0.67(0.33-1.37)$ \\
High TC & $<0.001$ & Reference & $8.12(4.40-15.00)$ & $1.76(0.88-3.54)$ \\
Abnormal AST & 0.629 & Reference & $0.87(0.54-1.40)$ & $1.24(0.79-2.04)$ \\
Abnormal ALT & 0.002 & Reference & $2.25(1.49-3.41)$ & $1.29(0.84-1.96)$ \\
\hline
\end{tabular}

The conditional probabilities of positive response to having MS components are demonstrated in the second section of Table 3. The first class, named "non-MS," included $55.1 \%$ of the study participants and was characterized by individuals exhibiting low probability for having all MS components. The second class, at risk, included $21.3 \%$ of the participants and was characterized by persons who had high probability for having low HDL and high TG. The third class, MS, included $23.6 \%$ of the participants and was characterized by individuals exhibiting high probability of having high $\mathrm{BP}, \mathrm{WC}$, and $\mathrm{FBS}$.

The first row of Table 3 shows the probabilities of membership in each latent class. For example, latent class 1, Non-MS, included almost $55 \%$ of the participants and latent class 3 , MS, included $23.6 \%$ of the participants.

Table 4 shows the odds ratios of membership in each latent class. For simplicity of interpretation, the first class (non-MS) was considered as a reference class, and membership in latent classes 2 and 3 was calculated compared to latent class 1 . This table indicates that having high LDL decreases the odds of membership in class 2 compared to class 1. Also, having high TC increases the odds of membership in class 2 compared to class 1 . This table shows that having abnormal AST have no significant effect on membership of participants in different classes compared to the first class.

\section{Discussion}

In this study, we identified latent subgroups of adults based on metabolic syndrome components using LCA and we were able to detect 3 separate classes: non-MS, at risk, and MS. The probability of engaging in each component of metabolic syndrome was low in the first class (non-MS). In the second class (at risk), however, the probability of having high HDL and high TG was high. In latent class 3 (MS), the probability of having high blood pressure, high abdominal obesity, and high FBS was high. There are 2 main groups of analysis in quantitative research: variable-centered and person-centered. Variable-centered analysis includes correlations, ANOVAs, path models, and regressions, which only assess the relationship between variables. Person-centered analysis includes cluster analysis and latent class analysis, which are used to investigate how variables are combined across individuals. Unlike variablecentered analysis that determines how characteristics are related to each other, person-centered analysis investigates how these variables group within individuals. As a result, LCA (as a person-centered analysis) can facilitate targeting future intervention resources to subgroup of adults based on MS components that promise to show the maximum treatment response $(25,26)$.

In this study, only 1 latent class (the third class) had high probability for at least 3 components of MS consistently. In other words, the prevalence of metabolic syndrome was estimated as nearly $24 \%$ with LCA. On the other hand, about $21 \%$ of the participants were at risk for developing metabolic syndrome (the second class). The third class (MS) may indicate a clinical pathway generating the observed features of metabolic syndrome. From prevention view, the policymakers should focus on blood pressure, abdominal obesity, and fast blood sugar for reducing the probability of engaging in cardiovascular diseases, type 2 diabetes, and other complications of metabolic syndrome. In the second class, low HDL and high TG cooccurred among $21 \%$ of the participants. Understanding how the metabolic syndrome components comorbid and cluster together in the latent classes 2 and 3 can help clinicians to interpret the pathophysiology of metabolic syndrome. A similar study among Iranian adults showed that abdominal obesity had an important role in classifying the study samples (20). In the present study, we found that some variables have high probability only in 1 class. It seems that for prevention programs, all these variables should have same importance. Nonetheless, results indicated that interventions for reducing the prevalence of blood pressure, abdominal obesity, and fasting blood sugar should be in priority, because of the high prevalence of latent class 3 compared to class 2 . The most important finding in LCA model was that among persons in latent class 3, some components of metabolic syndrome (low HDL and high TG) did not play an influential role in classifying the participants. On the other hand, these factors have high probability in the second class. In other words, about $45 \%$ of the study sample engaged with 2 or 3 components of MS. The participants of this study had different patterns of MS components. Nevertheless, based on the results of this study, it seems that integrated interventional programs should be designed to reduce the prevalence of all components of MS.

Only a few published studies used LCA to identify the latent classes of metabolic syndrome components. In addition, the researchers have used different criteria to specify subgroups. Also, the study samples were different among similar studies.

Edward et al found 3 latent classes of metabolic syndromes among nondiabetic individuals. They showed that only 1 latent class was strongly associated with all MS components. Moreover, they found another latent class that was associated with hyperglycemia and hypertension (19).

Another study indicated that among participants of the multiethnic study of atherosclerosis (MESA) participants, there were 3 latent classes of MS components. The authors named them as non-MS, low risk, and MS. They found that $29.9 \%$ and $35.4 \%$ of females and males were in latent class, respectively (18). 
Abbasi-Ghahramanloo et al identified 4 latent classes of MS in Iranian adults: (1) non-MS (38.4\%), (2) low-risk (18.6\%), (3) high-risk (24.2\%), and (4) MS (18.7\%). In other words, in this study, about $19 \%$ of the participants had a low-risk and 24\% a high-risk of developing MS (20). In comparison to Abbasi-Ghahramanloo et al study, our findings indicated that $21.3 \%$ of the participants were at risk of MS and 23.6\% were engaged with MS. The best reason for this difference could be related to gender of the participants. Our study focused on men only.

Prior studies stated that triglyceride (TG) is an independent risk factor in developing CVD (27). On the other hand, it proposed that increased TG production in the liver due to insulin resistance preferentially produces TG-rich very low-density lipoprotein $=\mathrm{s}$ (VLDL), which finally generates small dense-LDL particles (28). The association of TG concentration and LDL has been shown in a variable-centered study (29). In the present person-centered study, the results showed that having high TG increases the odds of membership in the second class and having high LDL significantly reduces the odds of membership in the second class $(\mathrm{OR}=0.27,95 \% \mathrm{CI}=0.13-0.56)$. In the second class, the probability of having low HDL is high too and the effect of high LDL is not related only to high TG.

The present study indicated that having abnormal ALT increases the odds of membership in latent class 2 (OR $=$ $2.25,95 \% \mathrm{CI}=1.49-3.41)$. ALT as a marker of liver injury have been associated with dyslipidemia, including triacylglycerols and low HDL (30-33). Our study also showed that abnormal ALT had no significant effect on the third latent class. Previous findings indicated that the ALT level is associated with gender of the participants and body mass index in the general population (34). These 2 factors were not assessed in our study.

In this study, we identified latent classes of MS among a sample of men who were living in Qom for the first time. However, as a limitation, it was not clear how many people were unaware of their status or were not treated. Also, we were unable to subgroup and compare clustering of the participants in different demographic groups such as smokers versus nonsmokers and other relevant factors.

\section{Conclusion}

Our study showed the separated subgroups of MS components among a sample of Iranian men. Results indicated that notable percent of samples fell in "at risk" and "MS" classes, which stress the necessity of designing preventive interventions with considering risk factors for these specific stratums of population.

\section{Acknowledgments}

This study was supported by Research Vice-Chancellor of Qom University of Medical Sciences. The authors are thankful for this financial support.

\section{Conflict of Interests}

The authors declare that they have no competing interests.

\section{References}

1. Chandey M, Kaur S, Kaur H. Prevalence of metabolic syndrome in young adults: a study from North India. Int J Adv Med. 2017;4(2):463466.

2. Kaykhaei M, Hashemi M, Narouie B, Shikhzadeh A, Jahantigh M, Shirzaei E, et al. Prevalence of metabolic syndrome in adult population from zahedan, southeast iran. Iran J Public Health. 2012;41(2):70.

3. Dalvand S, Niksima SH, Meshkani R, Gheshlagh RG, Sadegh-Nejadi S, Kooti W, et al. Prevalence of metabolic syndrome among iranian population: A systematic review and meta-analysis. Iran J Public Health. 2017;46(4):456.

4. Nolan PB, Carrick-Ranson G, Stinear JW, Reading SA, Dalleck LC. Prevalence of metabolic syndrome and metabolic syndrome components in young adults: A pooled analysis. Prev Med Rep. 2017;7:211-215.

5. El Brini O, Akhouayri O, Gamal A, Mesfioui A, Benazzouz B. Prevalence of metabolic syndrome and its components based on a harmonious definition among adults in Morocco. Diabetes Metab. Syndr Obes Targets Ther. 2014;7:341.

6. Lone S, Lone K, Khan S, Pampori RA. Assessment of metabolic syndrome in Kashmiri population with type 2 diabetes employing the standard criteria's given by WHO, NCEPATP III and IDF. Clin Epidemiol Glob Health. 2017;7(4):235-239.

7. Moore JX, Chaudhary N, Akinyemiju T. Peer Reviewed: Metabolic Syndrome Prevalence by Race/Ethnicity and Sex in the United States, National Health and Nutrition Examination Survey, 1988-2012. Prev Chronic Dis. 2017;14.

8. Gill RM, Khan SA, Jackson RT, Duane M. Prevalence of the Metabolic Syndrome in Central and South American Immigrant Residents of the Washington, DC, Area. Med J Nutrition Metab. 2017;2017.

9. Scuteri A, Laurent S, Cucca F, Cockcroft J, Cunha PG, Mañas LR, et al. Metabolic syndrome across Europe: different clusters of risk factors. Eur J Prev Cardiol. 2015;22(4):486-491.

10. Harikrishnan S, Sarma S, Sanjay G, Jeemon P, Krishnan M, Venugopal K, et al. Prevalence of metabolic syndrome and its risk factors in Kerala, South India: Analysis of a community based crosssectional study. PloS One. 2018;13(3):e0192372.

11. Aljohani NJ. Metabolic syndrome: Risk factors among adults in Kingdom of Saudi Arabia. J Fam Commun Med. 2014;21(3):170.

12. Ferns GA, Ghayour-Mobarhan M. Metabolic syndrome in Iran: a review. Diabetes Metab Syndr. 2018.

13. Mazloomzadeh S, Khazaghi ZR, Mousavinasab N. The Prevalence of Metabolic Syndrome in Iran: A Systematic Review and Meta-analysis. Iran J Public Health. 2018;47(4):473.

14 Amirkalali B, Fakhrzadeh H, Sharifi F, Kelishadi R, Zamani F, Asayesh $\mathrm{H}$, et al. Prevalence of metabolic syndrome and its components in the Iranian adult population: a systematic review and metaanalysis. Iran Red Crescent Med J. 2015;17(12).

15. Mohammadbeigi A, Farahani H, Moshiri E, Sajadi M, Ahmadi R, Afrashteh S, et al. Prevalence of Metabolic Syndrome and Associations with Lipid Profiles in Iranian Men: A Population-Based Screening Program. World J Mens Health. 2018;36(1):50-56.

16. Chen S, Guo X, Yu S, Zhou Y, Li Z, Sun Y. Metabolic syndrome and serum liver enzymes in the general chinese population. Int $J$ Environ Res Public Health. 2016;13(2):223.

17. Elizondo-Montemayor L, Ugalde-Casas PA, Lam-Franco L, Bustamante-Careaga H, Serrano-González M, Gutiérrez NG, et al. Association of ALT and the metabolic syndrome among Mexican children. Obes Res Clin Pract. 2014;8(1):e79-e87.

18. Riahi SM, Moamer S, Namdari M, Mokhayeri Y, Pourhoseingholi MA, Hashemi-Nazari SS. Patterns of clustering of the metabolic syndrome components and its association with coronary heart disease in the Multi-Ethnic Study of Atherosclerosis (MESA): A latent class analysis. Int J Cardiol. 2018.

19. Boyko EJ, Doheny RA, McNeely MJ, Kahn SE, Leonetti DL, Fujimoto WY. Latent class analysis of the metabolic syndrome. Diabetes Res Clin Pract. 2010;89(1):88-93.

20. Abbasi-Ghahramanloo A, Soltani S, Gholami A, Erfani M, Yosaee S. Clustering and combining pattern of metabolic syndrome components among Iranian population with latent class analysis. Med J Islam Repub Iran. 2016;30:445.

21. Abbasi-Ghahramanloo A, Janani L, Malakouti SK, Rabetian M, Rimaz S. Risk taking behaviors in relation to Attention Deficit and 
Hyperactivity Disorder in Iranian male workers: a latent class analysis. Neuropsychiatr Dis Treat. 2019;15:2513.

22. Mohammadbeigi A, Moshiri E, Mohammadsalehi N, Ansari H, Ahmadi A. Dyslipidemia prevalence in Iranian adult men: the impact of population-based screening on the detection of undiagnosed patients. World J Mens Health. 2015;33(3):167-173.

23. Expert Panel on Detection E. Executive summary of the third report of the National Cholesterol Education Program (NCEP) expert panel on detection, evaluation, and treatment of high blood cholesterol in adults (Adult Treatment Panel III). Jama. 2001;285(19):2486.

24. Afrashteh S, Ghaem H, Gholami A, Tabatabaee HR, AbbasiGhahramanloo A. Cigarette smoking patterns in relation to religiosity and familial support among Iranian university students: A Latent Class Analysis. Tob Induc Dis. 2018;16(July).

25. Howard MC, Hoffman ME. Variable-centered, person-centered, and person-specific approaches: where theory meets the method. Organ Res Methods. 2018;21(4):846-876.

26. Lanza ST, Rhoades BL. Latent class analysis: an alternative perspective on subgroup analysis in prevention and treatment. Prev Sci. 2013;14(2):157-168.

27. Harchaoui KEL, Visser ME, Kastelein JJP, Stroes ES, Dallinga-Thie GM. Triglycerides and cardiovascular risk. Curr Cardiol Rev. 2009;5(3):216-222.

28. Hirano T. Metabolic syndrome and small dense LDL-cholesterol. Rinsho byori. Jpn J Clin Onco. 2007;55(5):434-438.

29. Cho Y, Lee SG, Jee SH, Kim JH. Hypertriglyceridemia is a major factor associated with elevated levels of small dense LDL cholesterol in patients with metabolic syndrome. Ann Lab Med. 2015;35(6):586594.

30. Marchesini G, Brizi M, Bianchi G, Tomassetti S, Bugianesi E, Lenzi $\mathrm{M}$, et al. Nonalcoholic fatty liver disease: a feature of the metabolic syndrome. Diabetes. 2001;50(8):1844-1850.

31. Kelley DE, McKolanis TM, Hegazi RA, Kuller LH, Kalhan SC. Fatty liver in type 2 diabetes mellitus: relation to regional adiposity, fatty acids, and insulin resistance. Am J Physiol Endocrinol Metab. 2003;285(4):E906-E916.

32. Burgert TS, Taksali SE, Dziura J, Goodman TR, Yeckel CW, Papademetris X, et al. Alanine aminotransferase levels and fatty liver in childhood obesity: associations with insulin resistance, adiponectin, and visceral fat. J Clin Endocrinol Metab. 2006;91(11):4287-4294.

33. Bethel M, Deedwania P, Levitt N, Schmitz O, Huntsman-Labed A, Califf R, et al. Metabolic syndrome and alanine aminotransferase: a global perspective from the NAVIGATOR screening population. Diabet Med. 2009;26(12):1204-1211.

34. Vernon G, Baranova A, Younossi Z. Systematic review: the epidemiology and natural history of non-alcoholic fatty liver disease and non-alcoholic steatohepatitis in adults. Aliment Pharmacol Ther. 2011;34(3):274-285. 